\title{
Large-vessel Giant Cell Arteritis: A Rare Cause of Acute Upper Limb Ischemia - Case Presentation and Review of the Literature
}

\author{
Katalin Makó, Corina Ureche, Emőke Horváth \\ University of Medicine, Pharmacy, Science and Technology, Târgu Mureș, Romania
}

\begin{abstract}
Introduction: Acute upper extremity ischemia is an uncommon vascular emergency due to a relatively rich collateral network and low workload of the upper limb. Its consequences depend on the site and etiology of the arterial occlusion. Case presentation: Aiming to emphasize the emerging role of Doppler ultrasound in the diagnosis of acute upper limb ischemia, we report the case of a 70-year-old female, with severe left arm resting pain and digital cyanosis. Due to the patient's age and the presence of cardiovascular risk factors, cardioembolic or thrombotic arterial occlusion would have been the most likely diagnosis in this case, but the color Doppler ultrasound revealed severe left axillary arterial stenosis with hypoechoic wall swelling, being highly suggestive for arteritis. Temporal artery biopsy was performed, which confirmed giant cell arteritis. An excellent clinical response was obtained after initiation of treatment. Conclusion: In acute upper limb ischemia, color duplex ultrasound provides quick information about the etiology and localization of arterial lesions, offering characteristic findings in case of large-vessel giant cell arteritis.
\end{abstract}

Keywords: acute upper limb ischemia, giant cell arteritis

\section{ARTICLE HISTORY}

Received: September 7, 2019

Accepted: November 30, 2019

\section{CORRESPONDENCE}

Katalin Makó

Department of Internal Medicine University of Medicine, Pharmacy, Science and Technology of Târgu Mureș Str. Gheorghe Marinescu nr. 38 540139 Târgu Mureș, Romania Tel: +40 265212886

E-mail: makokatalin@yahoo.com

\section{INTRODUCTION}

Acute limb ischemia is defined as an abrupt reduction in arterial perfusion, usually producing new or worsening symptoms and signs, and it may potentially threat the viability of the affected limb. Generally, initial presentation is up to 2 weeks following the acute event. ${ }^{1}$ The sudden occlusion of the upper limb arteries can be produced by various pathologies. The main cause of acute upper limb ischemia is a cardioembolic occlusion in patients with atrial fibrillation. Arterial thrombosis resulting from atherosclerotic plaque erosion or ulceration can also generate acute occlusion. Many other cardiac and non-cardiac disorders have been identified as possible causes of upper limb acute ischemia, including arterial thoracic outlet syndrome, traumatic or iatrogenic occlusion, arterial aneurysm, fibromuscular dysplasia, arteritis, thrombophilia, essential thrombocytosis. ${ }^{2}$ From a clinical point of view, acute upper limb ischemia represents a vascular emergency, and its prompt diagnosis and adequate treatment is mandatory for the prevention of dramatic complications.

Giant cell arteritis (GCA) is the most frequent form of systemic vasculitis located at the medium and large vessels, with a predilection for the cranial branches of the carotid artery, typically affecting adults over 50 years of age. Clinically, it is characterized by a wide spectrum of 


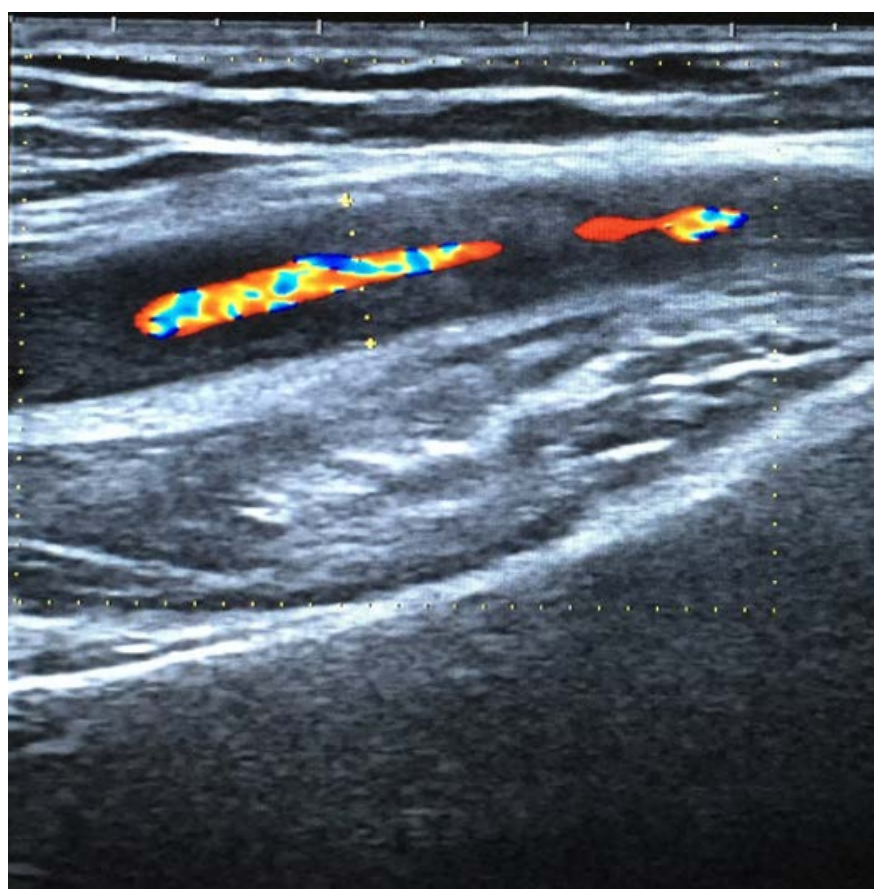

FIGURE 1. Left axillary artery ultrasound, longitudinal view, before treatment. Hypoechoic wall swelling (halo), size $1.8 \mathrm{~mm}$.

symptoms secondary to the involvement of the cranial arteries: headaches, visual loss, stroke, and jaw claudication. The prevalence of extracranial large vessel involvement (LV-GCA) was traditionally estimated at 3-15\%, if only the cardinal GCA symptoms are considered. Autopsy studies on GCA patients have demonstrated histological signs of large-vessel involvement in $80 \%$ of patients, and imaging studies revealed extensive radiographic involvement of the aorta and its major branches in up to $83 \%$ of patients. ${ }^{3,4}$

\section{CASE PRESENTATION}

A 70-year-old Caucasian overweight woman, with known arterial hypertension, presented with severe rest pain in the left arm, coldness, pallor, and paresthesia of the left hand, and digital cyanosis. She had no other symptoms (e.g., headache, jaw claudication, malaise, fever, or visual disturbances). The patient described worsening left shoulder pain for several months, which had been interpreted as shoulder osteoarthritis by her general practitioner (GP) and was treated with non-steroid anti-inflammatory drugs without results. Clinically, she had cold and cyanotic hands with impalpable radial and cubital pulses, and no measurable blood pressure on the left arm. Continuous wave (CW) Doppler examination revealed a systolic pressure of $140 \mathrm{mmHg}$ in the right ulnar and radial arteries, and of only $30 \mathrm{mmHg}$ in the left ulnar and radial arteries.
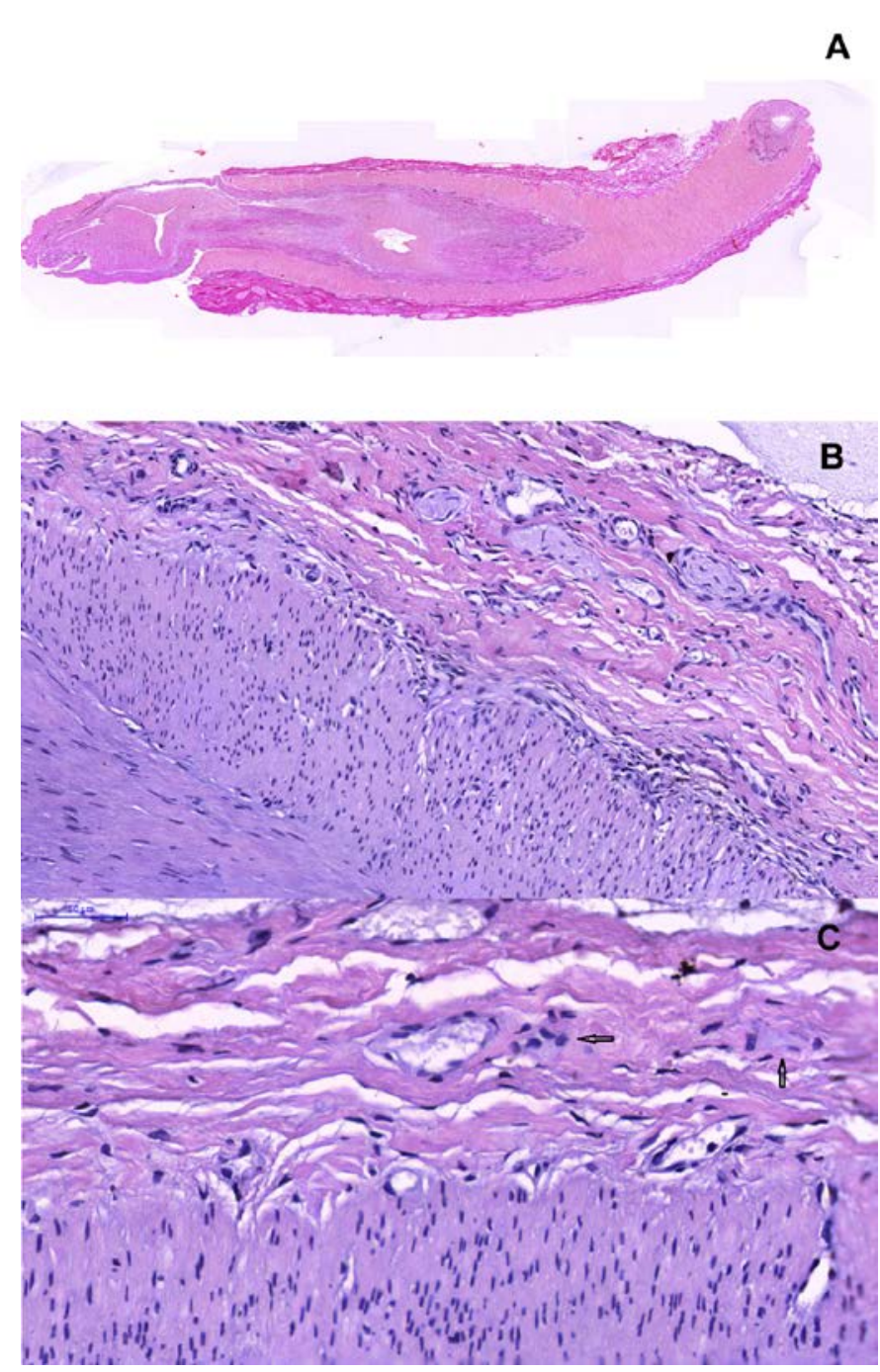

FIGURE 2. Narrowed vessel lumen with irregularly thickened intima and fibrotic media associated with disrupted elastic lamina (A - Elastic stain, 0.5 magnification). In the thickness of the wall there is a mild chronic inflammatory infiltrate (B - H\&E stain, 4.5 magnification). The giant cells (arrows) and rare macrophages adjacent vasa vasorum are constantly present (C - H\&E stain, 10 magnification).

Due to the patient's age and the presence of cardiovascular risk factors, atherosclerotic upper limb arterial disease would have been the most likely diagnosis in this case. However, the color duplex sonography (CDS) examination revealed a hypoechoic concentric rim of wall swelling (halo) around the lumen of the left axillary artery (AXA), with turbulent flow and localized increased flow velocity $(210 \mathrm{~cm} / \mathrm{s})$ (Figure 1). In longitudinal view, the halo size was $1.8 \mathrm{~mm}$. On the contralateral axillary, subclavian, carotid, and femoral arteries, only minimal atherosclerotic wall alterations were detected.

At the level of the temporal arteries (TA), clinically, no tenderness or constitutional symptoms were observed. 


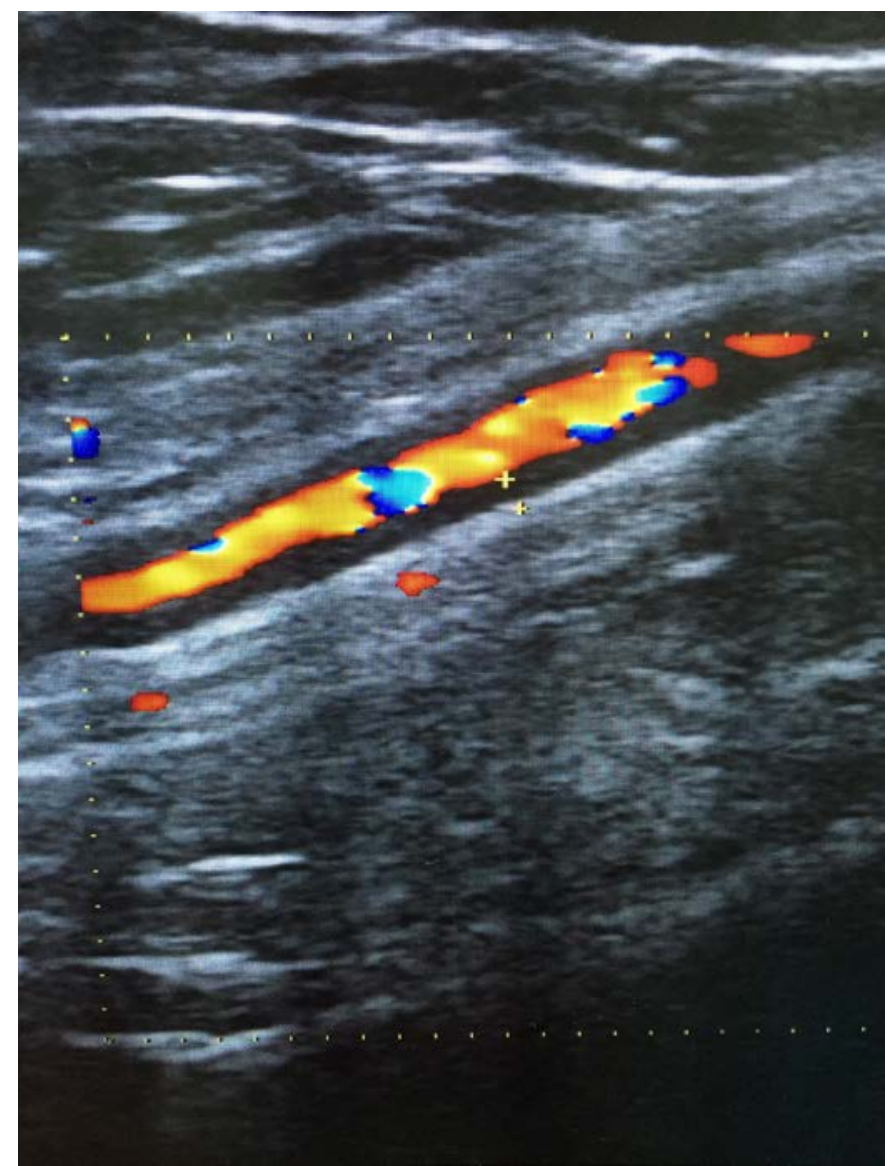

FIGURE 3. Left axillary artery ultrasound, longitudinal view, at 10 weeks of treatment. Hypoechoic wall swelling (halo), size $1.1 \mathrm{~mm}$.

The CDS of the temporal arteries revealed absence of flow and hypoechoic material in the right common superficial $\mathrm{TA}$, and the presence of a homogenous, hypoechoic circumferential wall thickening (halo size $0.8 \mathrm{~mm}$ ) in the left TA. The arteriography described smooth, concentric wall thickening of the left AXA with severe stenosis; no involvement of the subclavian artery, carotid artery, or the ascending aorta was detected.

The erythrocyte sedimentation rate was $121 \mathrm{~mm} / \mathrm{h}$; she had normochromic anemia, positive $\mathrm{C}$-reactive protein (CRP), and a negative infectious and autoimmune workup. Chest radiograph was normal, with no evidence of cervical ribs or chronic aortic dissection. The 12-lead ECG revealed normal sinus rhythm.

TA biopsy was performed, which revealed a narrowed vessel lumen with irregularly thickened intima, a fibrotic media associated with disrupted elastic lamina, mild chronic inflammatory infiltrate, and giant cells confirming GCA (Figure 2).

On the basis of the CDS finding, positive TA biopsy, and arteriography results, as well as on the systemic inflammatory response, a diagnosis of extracranial GCA was es- tablished. High-dose glucocorticoid therapy $(0.75 \mathrm{mg} / \mathrm{kg}$ per day prednisolone) was started. The patient was also started on clopidogrel (75 mg OD), due to increased risk of cardiovascular events, and proton pump inhibitors (pantoprazole $40 \mathrm{mg} \mathrm{OD}$ ). The evolution was clinically favorable; the erythrocyte sedimentation rate was $43 \mathrm{~mm} / \mathrm{h}$ at 2 weeks and $10 \mathrm{~mm} / \mathrm{h}$ at 10 weeks after initiation of treatment. The follow-up CDS performed at 16 weeks revealed reduced swelling in the AXA wall (the halo size was $1.1 \mathrm{~mm}$ ) (Figure 3).

\section{DISCUSSIONS}

Recognizing giant-cell arteritis as the cause of unilateral acute upper limb ischemia may be challenging, especially when typical symptoms of GCA (headache, fever, visual loss, polymyalgia rheumatica) are missing. Large-vessel involvement in patients diagnosed with GCA has been found in more than $80 \%$ of patients, as shown via imaging studies. The most affected locations in case of LV-GCA are the thoracic aorta (45-65\%) and the subclavian arteries/AXAs (30-75\%). Positron emission tomography (PET) studies have shown that inflammation of the subclavian and axillary arteries is virtually always accompanied by thoracic aortitis. The involvement of large vessels is paramount because these patients present a high risk of developing thoracic aortic aneurysms, which in turn lead to increased mortality rates. ${ }^{3,4}$

\section{CLINICAL AND LABORATORY FEATURES}

GCA may present variable clinical signs depending on the affected arterial segment. In case of cranial GCA, classical signs and symptoms include headache, temporary artery tenderness and swelling, jaw claudication, or visual disturbances. ${ }^{3}$ These classical signs are generally absent in patients with LV-GCA. In these patients, limb claudication, pulse disturbances, vascular bruits, and aortic regurgitation murmur may appear. In addition, studies describe a younger age of disease onset, stronger female predominance, and longer time to diagnosis in case of patients with LV-GCA. Other common signs and symptoms of GCA and LV-GCA include fever of unknown origin, weight loss, malaise, elevated erythrocyte sedimentation rate, normochromic anemia, positive CRP, and a negative infectious and autoimmune workup. In some cases, overlapping phenotypes may be observed. ${ }^{4}$ The characteristic clinical signs and symptoms of LV-GCA and GCA are presented in Table 1.

Vasculitis should always be part of the differential diagnosis in large artery obstruction, even in patients with 
TABLE 1. Clinical signs and symptoms of LV-GCA and GCA $4,18-20$

\begin{tabular}{lcc}
\hline Sign and symptoms & LV-GCA & GCA \\
\hline Headache & $+/-$ & ++ \\
Visual symptoms & - & ++ \\
Temporal artery tenderness/swelling & $+/-$ & ++ \\
Fever & + & + \\
Weight loss & $+/-$ & $+/-$ \\
Jaw claudication & - & + \\
Tongue pain & - & + \\
Limb claudication & ++ & - \\
Blood pressure difference & ++ & - \\
Vascular bruits & + & - \\
Elevated ESR & ++ & ++ \\
Elevated CRP & + & + \\
\hline
\end{tabular}

multiple cardiovascular risk factors. Vascular inflammation must be differentiated from atherosclerotic changes in patients over 50 years. The two disorders present different locations (atherosclerosis being more common in the iliac, femoral, common and internal carotid arteries) and morphology of lesions. ${ }^{4}$ As stated in the 2017 ESC Guidelines, in case of upper limb ischemia, the differential diagnosis should include atherosclerosis, thoracic outlet syndrome, arteritis, radiation artery fibrosis, embolic origin, and fibromuscular dysplasia. ${ }^{1}$

\section{THE ROLE OF COLOR DUPLEX SONOGRAPHY IN THE DIAGNOSIS OF LV-GCA}

The development of noninvasive imaging methods (CDS, magnetic resonance angiography, computed tomography angiography, PET) has significantly improved the diagnosis of LV-GCA. However, a consensus statement on the diagnostic criteria of large-vessel involvement as well as the modality and timing of evaluation is lacking.

A case-control study that included subjects with newonset GCA has identified large-vessel involvement in 29\% of cases, by using CDS. ${ }^{5}$ Large-vessel involvement was also noted in $30 \%$ of 176 patients with GCA, with the use of CDS for imaging evaluation of the subclavian, axillary, proximal brachial, and temporal arteries. ${ }^{6}$

Color duplex sonography is the most accessible diagnostic tool for the rapid diagnosis of LV-GCA, this method being supported by increasing robust evidence. ${ }^{7-9}$ The benefits of CDS include its wide accessibility, the absence of ionization radiations, and low costs. However, the method is characterized by relatively high inter-reader variability;
TABLE 2. Ultrasonographic findings in $L V-G C A^{8-10}$

\begin{tabular}{ll} 
Ultrasonographic sign & Description \\
\hline Halo sign & $\begin{array}{l}\text { Hypoechoic, homogenous wall swelling, } \\
\text { commonly concentric, visible both in longi- } \\
\text { tudinal and transverse scans }\end{array}$ \\
Maximum halo size & $\begin{array}{l}\text { Measured in longitudinal view in mm } \\
\text { Cut-off values: } 1.5-2 \text { mm in the AXA, } \\
\text { o.5-0.8 mm in the TA }\end{array}$ \\
The halo does not disappear after applying \\
pressure with the transducer, even if the \\
vessel lumen remains occluded (in TAs) \\
Stenosis
\end{tabular}

therefore, the evaluation of large vessels should be performed by experienced ultrasonographers. The halo sign is the most important and typical ultrasonographic finding in vasculitis, being a hypoechoic homogeneous area easily identifiable between the vessel lumen and the perivascular tissue. ${ }^{7,8}$ In GCA, edema and cell infiltrates are located in the media and extend to the intima; the ultrasound detects this edematous parietal change as a hypoechoic material around the artery lumen that contrasts to the surrounding tissues. It can be circumferential or eccentric in axial views and may be confirmed by a positive "compression sign" in the temporal arteries and its branches, meaning that the halo does not disappear after applying pressure with the transducer, even if the vessel lumen remains occluded..$^{8,9}$ The most important ultrasonographic findings in LV-GCA are presented in Table 2.

A recent prospective study published by Schaffer et al. has established that normal intima-media thickness (IMT) is approximately $0.2 \mathrm{~mm}$ in the TA and $0.6 \mathrm{~mm}$ in the AXA. Vasculitis changes commonly result in $0.5-0.8 \mathrm{~mm}$ wall swelling in TAs and $1.5-2 \mathrm{~mm}$ in AXAs. ${ }^{10}$

Czihal et al. aimed to determine the diagnostic accuracy of CDS of the TAs and AXAs for the diagnosis of GCA. Their results revealed that a cut-off value of $\geq 1.2 \mathrm{~mm}$ in intima media thickness evaluated in the AXA offers a sensitivity of $81.3 \%$ and a specificity of $96.1 \%$ for the diagnosis of LV-GCA. ${ }^{11}$

The presence of a halo on CDS is considered a sign of disease activity. In the TAs, this hypoechoic wall swelling may resolve within a few days after glucocorticoid treatment initiation, but AXAs may take 1-2 months. ${ }^{8}$ Long-term monitoring of disease activity is important in 
patients with LV-GCA given the risk for vascular complications. However, consensus guideline recommendations on the method, duration, and timing of follow-up of these patients are lacking. ${ }^{4}$

\section{THE ROLE OF OTHER IMAGING TECHNIQUES IN THE DIAGNOSIS OF LV-GCA}

Medical and vascular surgical guidelines recommend baseline computed tomography angiography (CTA) or magnetic resonance angiography (MRA) evaluation in all patients with GCA or LV-GCA in order to evaluate the presence and extension of aortic involvement. ${ }^{12} \mathrm{~A}$ prospective study conducted by Prieto-Gonzalez et al. has evaluated the presence of large-vessel involvement in newly diagnosed GCA, as assessed by CTA, and has detected signs of large-vessel vasculitis in $67.7 \%$ of cases. The involved vessels were as follows: aorta (65\%), brachiocephalic trunk $(47.5 \%)$, carotid arteries (35\%), subclavian arteries $(42.5 \%)$, and AXAs $(17.5 \%){ }^{13}$ Several retrospective and cross-sectional imaging studies have shown the presence of structural damage in the aortic wall in $10-33 \%$ of patients with GCA in a follow-up period of 10 years. ${ }^{14,15}$ The 18F-fluorodeoxyglucose (18FDG)-PET evaluation of GCA patients has demonstrated vascular hypermetabolism as a sign of vascular inflammation in large elastic arteries in up to $85 \%$ of cases. ${ }^{16}$ This method can detect early therapeutic response in patients with GCA, being a useful tool for assessment of disease activity. ${ }^{8}$

\section{THERAPEUTIC APPROACH OF PATIENTS WITH LV-GCA}

Studies comparing glucocorticoid effects and doses or immunosuppressive therapeutic regimens, especially for LV-GCA, are missing. Generally, subjects with signs of LV-GCA should be prescribed the same treatment regimen as patients with cranial GCA. The goal of treatment in patients with LV-GCA is rapid disease control by suppressing systemic and vascular inflammation, thus preventing ischemic organ damage.

Glucocorticoids (GC) are the treatment of choice for inducing remission in subjects with LV-GCA. Initial doses of $0.75-1.0 \mathrm{mg} / \mathrm{kg} / \mathrm{day}$, followed by gradual tapering is the regimen of choice. ${ }^{4}$ A prospective study evaluating the early outcomes of standardized GC treatment in biopsy- and CTA-proven LV-GCA has revealed a decrease in inflammation at the level of the large vessels, as demonstrated by a decreased number of affected segments and a decreased wall thickness, after a follow-up time of 13.5 months. ${ }^{17}$ The symptoms of GCA generally respond quickly to high-dose GCs with rapid reduction of inflammatory markers. GC dose-reduction should be considered in the absence of clinical symptoms and laboratory abnormalities (erythrocyte sedimentation rate, CRP). Patients should be monitored for evidence of relapse, in which case adjuvant therapy, such as methotrexate or other immunosuppressant, must be considered. ${ }^{18-20}$

\section{CONCLUSIONS}

Large-vessel involvement in GCA is a frequent disorder, and a multidisciplinary approach with a standardized CDS assessment of different vascular territories is needed to prevent the delay in diagnosis and consequent vascular complications. CDS is the most accessible tool for the rapid diagnosis of LV-GCA, this method being supported by increasing robust evidence. Considering that atypical localizations of the disease are common, the routine use of duplex ultrasound in patients with clinical signs of upper limb ischemia may lead to fewer cases of delayed or missed diagnosis.

\section{CONFLICT OF INTEREST}

The authors declare no conflict of interest.

\section{CONSENT FOR PUBLICATION}

Written informed consent was obtained from the patient for publication of this case report and any accompanying images, and the publication of the case was approved by the ethics committee of the institution.

\section{REFERENCES}

1. Aboyans V, Ricco JB, Bartelink MEL, et al. 2017 ESC Guidelines on the Diagnosis and Treatment of Peripheral Arterial Diseases, in collaboration with the European Society for Vascular Surgery (ESVS): Document covering atherosclerotic disease of extracranial carotid and vertebral, mesenteric, renal, upper and lower extremity arteries Endorsed by: the European Stroke Organization (ESO)The Task Force for the Diagnosis and Treatment of Peripheral Arterial Diseases of the European Society of Cardiology (ESC) and of the European Society for Vascular Surgery (ESVS). Eur Heart J. 2018;39:763816. doi: 10.1093/eurheartj/ehx095.

2. Chisari A, Pistritto AM, Bellosta R, et al. Upper limb ischemia from arterial thromboembolism: a comprehensive review of incidence, etiology, clinical aspects, diagnostic tools, treatment options and prognosis. Minerva Cardioangiol. 2016;64:625-634

3. Jennette JC, Falk RJ, Bacon PA, et al. 2012 revised International Chapel Hill Consensus Conference Nomenclature of 
Vasculitides. Arthritis Rheum. 2013;65:111. doi: 10.1002/ art.37715.

4. Koster MJ, Matteson EL, Warrington KJ. Large-vessel giant cell arteritis: diagnosis, monitoring and management. Rheumatology. 2018;57:ii32-ii42. doi: 10.1093/rheumatology/ kex424.

5. Ghinoi A, Pipitone N, Nicolini A, et al. Large-vessel involvement in recent-onset giant cell arteritis: a case-control colourDoppler sonography study. Rheumatology. 2012;51:730-734. doi: 10.1093/rheumatology/ker329.

6. Schmidt WA, Seifert A, Gromnica-Ihle E, et al. Ultrasound of proximal upper extremity arteries to increase the diagnostic yield in large-vessel giant cell arteritis. Rheumatology. 2008;47:96-101. doi: 10.1093/rheumatology/kem322.

7. Schmidt WA, Natusch A, Moller DE, et al. Involvement of peripheral arteries in giant cell arteritis: a color Doppler sonography study. Clin Exp Rheumatol. 2002;20:309-318.

8. Schmidt WA. Role of ultrasound in the understanding and management of vasculitis. Ther Adv Musculoskelet Dis. 2014;6:39-47. doi: 10.1177/1759720X13512256.

9. Monti S, Floris A, Ponte C, et al. The use of ultrasound to assess giant cell arteritis: review of the current evidence and practical guide for the rheumatologist. Rheumatology. 2018;57:227-235. doi: 10.1093/rheumatology/kex173.

10. Schafer VS, Juche A, Ramiro S, et al. Ultrasound cut-off values for intima-media thickness of temporal, facial and axillary arteries in giant cell arteritis. Rheumatology. 2017;56:14791483. doi: 10.1093/rheumatology/kex143.

11. Czihal M, Schrottle A, Baustel K, et al. B-mode sonography wall thickness assessment of the temporal and axillary arteries for the diagnosis of giant cell arteritis: a cohort study. Clin Exp Rheumatol. 2017;35:128-133.

12. Borchers AT, Gershwin ME. Giant cell arteritis: a review of classification, pathophysiology, geoepidemiology and treatment. Autoimmun Rev. 2012;11:A544-A554. doi: 10.1016/j. autrev.2012.01.003.
13. Prieto-Gonzalez S, Arguis P, Garcia-Martinez A, et al. Large vessel involvement in biopsy-proven giant cell arteritis: prospective study in 40 newly diagnosed patients using CT angiography. Ann Rheum Dis. 2012;71:1170-1176. doi: 10.1136/ annrheumdis-2011-200865.

14. Gonzalez-Gay MA, Garcia-Porrua C, Pineiro A, et al. Aortic aneurysm and dissection in patients with biopsy-proven giant cell arteritis from northwestern Spain: a populationbased study. Medicine. 2004;83:335-341. doi: 10.1097/01. md.0000145366.40805.f8.

15. Garcia-Martinez A, Arguis P, Prieto-Gonzalez S, et al. Prospective long term follow-up of a cohort of patients with giant cell arteritis screened for aortic structural damage (aneurysm or dilatation). Ann Rheum Dis. 2014;73:1826-1832. doi: 10.1136/annrheumdis-2013-203322.

16. Blockmans D, de Ceuninck L, Vanderschueren S, et al. Repetitive $18 \mathrm{~F}$-fluorodeoxyglucose positron emission tomography in giant cell arteritis: a prospective study of 35 patients. Arthritis Rheum. 2006;55:131-137. doi: 10.1002/art.21699.

17. Prieto-Gonzalez S, Garcia-Martinez A, Tavera-Bahillo I, et al. Effect of glucocorticoid treatment on computed tomography angiography detected large-vessel inflammation in giantcell arteritis. A prospective, longitudinal study. Medicine. 2015;94:e486. doi: 10.1097/MD.0000000000000486.

18. Mukhtyar C, Guillevin L, Cid MC, et al. EULAR recommendations for the management of large vessel vasculitis. Ann Rheum Dis. 2009;68:318-323. doi: 10.1136/ard.2008.088351.

19. Dasgupta B, Borg FA, Hassan N, et al. BSR and BHPR guidelines for the management of giant cell arteritis. Rheumatology. 2010;49:1594-1597. doi: 10.1093/rheumatology/keq039a.

20. Bienvenu B, Ly KH, Lambert M, Agard C, et al. Management of giant cell arteritis: recommendations of the French study Group for Large Vessel Vasculitis (GEFA). Rev Med Interne. 2016;37:154-165. doi: 10.1016/j.revmed.2015.12.015. 